\title{
The challenges of COVID-19-related weight gain in children
}

\author{
Mira FLOREA ${ }^{1}$, Lucia LOTREAN ${ }^{1}$, Aida PUIA ${ }^{1}$, Ana Maria Alexandra STANESCU ${ }^{2}$, \\ Anca Angela SIMIONESCU ${ }^{3,4}$, Rodica CORNEAN ${ }^{5,6}$ \\ ${ }^{1}$ Community Medicine Department, "Iuliu Hatieganu" University of Medicine and Pharmacy, \\ Cluj-Napoca, Romania \\ ${ }^{2}$ Department of Family Medicine, "Carol Davila" University of Medicine and Pharmacy, Bucharest, Romania \\ ${ }^{3}$ Department of Obstetrics and Gynecology, \\ "Carol Davila" University of Medicine and Pharmacy, Bucharest, Romania \\ ${ }^{4}$ Department of Obstetrics and Gynecology, Filantropia Clinical Hospital, Bucharest, Romania \\ ${ }^{5}$ Pediatrics No.2, Clinical Emergency Hospital for Children, Cluj-Napoca, Romania \\ ${ }^{6}$ Department of Molecular Sciences-Medical Genetics, \\ "Iuliu Hatieganu" University of Medicine and Pharmacy, Cluj-Napoca, Romania
}

\begin{abstract}
The current epidemiological context with online schooling, inadequate physical activity and sedentary behavior in response to the COVID-19 pandemic may lead to an increased risk of childhood obesity. Many children in the developed world were already sedentary and 81\% of those aged 11-17 years were not physically active enough even before the pandemic, spending a lot of time using various electronic devices.

This paper focuses on the COVID-19-related weight gain of children and expert recommendations to alleviate its long- term detrimental consequences, based on data from several countries collected and published in 2020 in 2021. It revise data regarding weight gain in several countries during COVID-19 pandemic, factors which influence weight gain with a special attention to the pandemic context and challenges and opportunities for appropriate weight management of children during this difficult times.

Studies performed in developed countries have shown that the disruption of mealtimes, sleep habits and limited physical activity due to the pandemic have led to changes in children's food habits and an increased body weight among, being noticed several differences based on country, age, gender and socio-economical level.

We conclude on the need for an increased awareness among parents and healthcare professionals about the risk of children gaining body weight related to the life changes imposed by this long-lasting COVID-19 pandemic. Parent and child-based educational interventions, reset in this epidemiological context could positively influence children's behavior to resort to eating comfort before developing resistance to change and improve the recognition and management of their nutritional status.

Future research is needed to identify new educational strategies and school-based interventions for both children and parents, targeting an optimal and more active lifestyle to prevent childhood obesity.
\end{abstract}

Keywords: COVID-19 pandemic, disruption, mealtimes, sleep schedules, physical activity,

body weight gain 


\section{INTRODUCTION}

One year and a half into the "new normal" caused by the Coronavirus Disease 2019 (COVID-19) pandemic, parents and health professionals are worried about the considerable changes in the lives of children and adolescents. Despite the relatively low risk of developing severe disease in children infected with SARS-CoV-2 $[1,2]$, the COVID-19 pandemic induces health effects that extend beyond the infection itself. There are concerns that it will most likely exacerbate food insecurity and malnutrition among children in low- and middle-income countries [3] and, on the contrary, increase the silent epidemic of childhood obesity in upper-middle-income and high-income countries [4].

This pandemic found many children and adolescents in the developed world already sedentary, spending a lot of time using various technical devices. A study published in Lancet reported that, $81 \%$ of children aged 11-17 years were not physically active enough prior this crisis [5].

Online schooling, canceled or dramatically reduced physical activity classes, sports in parks, gyms restricted by the need for social distancing during the COVID-19 pandemic, lost routine, economic insecurity through parental job losses, disrupted meal routines and sleep habits have affected children and adults $[6,7]$. In this long-standing epidemiological context, one of the effects of the COVID-19 pandemic might be the weight gain of children.

This paper focuses on the COVID-19-related weight gain of children and expert recommendations to alleviate its long-term detrimental consequences, based on data from several countries collected and published in 2020 in 2021. It revise data regarding weight gain in several countries during COVID-19 pandemic, factors which influence weight gain with a special attention to the pandemic context and challenges and opportunities for appropriate weight management of children during this difficult times.

\section{WEIGHT GAIN DURING COVID-19 PANDEMIC AMONG CHILDREN IN SEVERAL COUNTRIES}

Weight increases with age among children and adolescents, consequently, the change in weight between different time points might be a physiological consequence of their physical development, being needed to use appropriate methods to assess if their weight is appropriate or not according to their age and height. World Health Organization, International Obesity Task Force and Center for disease control and prevention as well as other national organizations recommend tools for assessing overweight and obesity among children and adolescents based on their age and objective measured height and weight. Hence, the assessment of gain weight and its implication for the risk of development of overweight and obesity in children and adolescents is more difficult to be performed than in the case of adults [8]. Moreover, the lock down imposed during the pandemic decreased the possibility of objective measurements of weight and height and appropriate interpretation by trained health care personnel, the information being missing or reported data perceived by parents were used by several studies trying to assess weight gain among children and adolescents during COVID-19 pandemic [9-11].

A review performed on PubMed indexed studies published until 12 April 2021 concluded that there were performed several survey studies of lifestyle changes among children and a few with questions regarding weight gain, but no study using an objective measurement of weight gain among a broad pediatric population between pre- and post-lockdown eras (9). Since then, some studies were also using such an approach [9-11].

In a high-income country, Germany, a study performed after the first 6 months of pandemic among 1000 parents with children aged up to 14 years old showed that an increase of children's body weight during the pandemic was reported by $9 \%$ of parents, being noticed a greater prevalence $(23 \%)$ among children whose parents had a low level of school education ( $<10$ years), among those of 10-12 years old (19\%) and within this age group among boys ( $24 \%$ among boys vs $13 \%$ among girls) [4]. In addition, $27 \%$ of parents reported having gained weight since the start of the pandemic, without to be noticed differences based on gender, income or child age [4].

Data presented at the Annual Meeting of American Society of Nutrition Scientific Sessions, June 7-10, 2021 showed that one-third of parents who were surveyed in May 2020 and September 2020 regarding home food environment and child feeding practices reported that their children gained weight. The average weight gain was 9.6 pounds in those five months and parents reported being concerned about their children's nutritional status [9]. Parents' attention and interest in adequately recognizing their children's nutritional status has been the subject of studies over the past decades but currently, they are more than ever a priority [12-15].

A study from United States used electronic data records including objective measurement of height, weight and calculation of age and sex adjusted change in body mass index in September 2020 comparative to prior years among 6-17 years old children. The results 
showed that children's rate of unhealthy weight gain increased notably during the COVID-19 pandemic and especially among children already vulnerable to unhealthy weight gain [10].

A study from Israel that included 229 children and adolescents aged 0-18 years revealed a total mean weight-percentile significantly higher following 7 weeks of strict lockdown and stay-at-home regulations than the previously ( 40.44 vs. 38.82 , respectively, $p=0.029$ ), the increase being higher among children younger than 6 years and among boys [11].

\section{FACTORS PROMOTING THE OBESOGENIC BEHAVIORS}

Many factors that have changed the children and families' lives during the pandemic promoted obesogenic behaviors.

Factors directly related to the pandemic epidemiological context $[3,4,6,9]$ :

- High unemployment rates, financial burdens leading to increases in families' and children's food insecurity

- Home schooling leading to the fact that children no longer received meals provided by the school as well as to a greater responsibility for parents to provide meals and snacks for their children, while trying to deal with their work duties

- Food accumulation or the storage of unhealthy foods, such as non-perishable processed foods, which are stable on the shelf

- Inappropriate access to nutritious food products and walking and sport facilities due to lock down periods

Factors related to the complex disruption in children routines and behavior during pandemic, leading to increase in body mass index (BMI) $[1,4,9,11,12,17]$ :

- Disrupted or altered sleep schedules

- Change in nutritional level of food and lack of structured mealtimes

- Lack of structured physical activity

- Misperceptions of children and parents about normal children's body weight and inappropriate nutritional education

- Increased anxiety in adolescents, depression and feelings of loss, even the feeling of sadness for the events they missed during the pandemic contributed to eating disorders, making them resort to eating comfort

Changes in eating habits and body weight occur shortly after the alteration of physical activity and eating behavior in response to COVID-19, which might lead to resistance to change, increased risk of obesity, diabetes and cardiovascular disease in children [16].
The pandemic restrictions have disrupted daily routines for many families. Not attending school, staying at home challenged children in the terms of nutrition, sleep duration and its quality and physical activity. By staying home for work, learning and socializing, children and their families go to bed later and wake up later, disrupting their normal schedule. Late nights' impact worsens daily and affects other aspects of children's lives: mealtimes, nutrition and physical activity. Children and adolescents who go to bed late are more likely to eat more, even at night, to eat comfortable foods, such as carbohydrates (chips, cookies, etc.), which provides a short burst of energy that causes them to stay awake later but let them feel still starving and gaining unhealthy weight. It is a cycle of self-defeating that is hard to stop without family intervention, coming with a healthy routine restoration. Additionally, while families keep stable foods on the shelf, they seem to buy junk food, processed products, and high-calorie comfort diets [17]. Predictions consistent with the culture and home facilities demonstrate that not attending school can lead to more eating foods, junk foods, and/or miss meals among children of school age $[6,18,19]$.

A study from Germany showed that after the first 6 months of pandemic changes in children's food habits were noticed with increased consumption of salty snacks (18\%), sweet snacks (20\%), and soft drinks (18\%), potatoes, pasta, rice, and pizza (16\%), higher proportions of school-age children aged 10 years and older and boys being involved in these behaviors. On the other hand, consumption of fruits and vegetables also increased among all age groups independent of gender, as a consequence of family meal preparation [4].

An inactive lifestyle has a great influence especially on urban children who lack access to places where they can sustain social distancing. Screen time in childhood is associated with overweight and obesity, due to sedentariness and snacking during screen viewing $[6,20]$.

In addition, some children's and parents' misperceptions about children's weight led to lack of parent-child communication about health issues, unhealthy practices for self-management of weight, delayed addressing to healthcare professionals and late interventions [12]. Abnormal weight gain in childhood is a long-standing problem, as several studies showed that childhood obesity is related to adulthood weight gain [4]. A recent study showed that an abnormal increase in weight at the age of five was significantly linked to an increase in body mass index and fat mass in middle age [21]. 


\section{RECOMMENDATIONS FOR PROMOTING HEALTHY EATING AND ACTIVE LIFESTYLE DURING COVID-19 PANDEMIC}

\section{The experts' recommendations for increasing healthy eating}

World Health Organization, as well as several international and national organizations promotes recommendations with regard to promotion of a balanced diet among children which supply them with appropriate energy, macronutrients and micronutrients in accordance with their age, development phase, lifestyle and physiological or pathological conditions [22]. Eating the right foods at the right time during the day having grate impact on children's weight gain or loss. Three meals a day, plus one or two snacks are recommended. Meals should be medium size, balancing mix of food groups, with limited to no sugar. The carbohydrates offered are whole grain, fruits, vegetables, and lean proteins. Drinks should be water or low-fat milk. It is not recommended combining or skipping meals as it sets up a dangerous cycle of spikes (energy) and tiredness throughout the day. The parent should be mindful of what their child drinks. There are many calories in soda, energy and fruit drinks and must be avoided and replaced with water, water infused with fruit, or seltzer water if the child misses the bubbles found in soda. Regular mealtimes are important, reducing frequent snacking $[22,23]$.

Healthy foods must be consistently available at home. The real decision point for healthy eating is what parents bring into the house. The supermarket shopping experience shows that the shelves containing flour, beans and rice are full, while the shelves containing ramen noodles, chips, biscuits, sugar flakes, soft drinks and ready-to-eat processed products are empty $[6,18]$.

The involvement of children with meal and snack preparation is an opportunity to discuss in the family the meals for the week, to encourage to eat vegetables and fruits, and can help prepare the meals they enjoy most [22]. Parents should not restrict foods from one child while allowing them for other family members. Meal preparation is a great way for parents to spend quality time with their children, making the meals preparations a social event. It is important to eat at least one meal a day as a family. This will help every member of the family to enjoy the food and company. Small changes can have a big impact and setting goals together may optimize the family's lifestyle [23].

Another way to influence children's motivation is to reinforce healthy sleep habits disrupted during the pandemic. The parents should ensure their children receive enough sleep at night. Elementary school chil- dren should be preparing for bed or reading by 8 p.m. Adolescents may stay up until 10 or 10.30 p.m., but they need time to decompress and relax their minds and bodies. The electronic devices (cell phones, tablets, computers) should be kept out of the bedroom as the beeping, text or alerts can disrupt critical phases of sleep cycle [20].

In addition to the challenges, there are also opportunities for parents, teachers, health professionals, as well as for decision makers to get involved in promoting healthy nutrition among children for the next uncertain period, which could lead to lockdown periods, home or hybrid schooling and recovery after the difficult time during the pandemic.

Health care professionals, school and health policy makers should help parents and children in this process by developing, implementing and evaluating several actions such as:

- Providing appropriate school based nutritional education delivered through face to face, online or hybrid activities

- Assuring medical help for parents in order to prevent, identify and manage correctly the overweight and obesity among their children, including through use of telemedicine if needed

- Assuring nutritional school meals and developing strategies for food delivery and helping scheme in order to prevent food insecurity among families with low socio-economical background

- Assuring channels for appropriate access to nutritional food products, including promoting of locally produced foods which is also important for the sustainable development

- Appropriate funding and long-term strategies for assuring nutritional education and nutritional counseling targeting children and parents

\section{Recommendations for an active lifestyle}

According to the recommendations of World Health Organization children should perform at least one hour of moderate physical activity every day [22].

The increasing capacity for distance learning in schools should consider the prioritization of physical education in this epidemiological context. Along with the content of the lessons sent online by the teachers, children need to be involved by providing diversified physical education programs. There are various exercise plans that can be adjusted to the home curriculum [24]. Children should be encouraged by both parents and teachers to select a different activity each day from a list of proposed indoor or outdoor activities. Children can quickly develop favorites that lead to long-term enjoyment and more active lifestyle. Some activities can be done alone, while others may be better with an "ac- 
tivity mate". Exercising with others creates responsibility and increases the chance of children remaining active [22].

Challenging them through competitions with family members, friends using fitness apps or active video games can further stimulate. Many researchers have recommended reducing sedentary activities, such as watching television and playing videogames, to prevent obesity among children and adolescents [25]. Some apps (applications) provide a workout plan with training (such as videos, photos, and explanatory text) and provide information about the number of sets and repetitions, and the rest time between different recommended exercises. In the last decade it has been proven that technology-based exercises, such as active video games, that require body mobility to perform are stimulating for children and can reduce screen time [26]. Video game manufacturers have created "active" video game consoles and introduced the term "exergames" to an effort to make this typically sedentary activity more physically engaging.

The term "exergame" is frequently used in the literature, media, and is a catchy word. Exergames are the combination of exertion and video games including strength training, balance, and flexibility activities. They supply some activities such as fitness, dance, martial arts, and various sports and offer gamers sporting experiences that mimic the real game or sport, but are not better than real exercise, and should not replace physical education [26]. Exergaming is playing exergames to promote physical activity. Some physical education teachers have embraced this technology in their classes to motivate children who show a lack of interest in traditional physical education activities. However, these exergames don't provide the same skill development as traditional physical education. While parents should encourage their children to play exer- games instead of sedentary video games during leisure time, expert do not recommend teachers replace traditional physical education with exergames when motor skill improvement is the primary goal of the lessons [26].

The families must be encouraged to seek advice from family doctor and child's pediatrician about their child weight concerns. They can offer weight management advice by in-person visits or through telemedicine [27]. There are currently over 300,000 health-related applications around the world that changed how patients interact with the healthcare professionals. They need to be evaluated, validated and effectively disseminated into routine clinical practice [28].

\section{CONCLUSIONS}

Public health policy strategies should be geared towards promoting physical activity, and more efforts are required to develop interventions that target the highrisk groups of overweight and obese children prior the pandemic.

We conclude on the need for an increased awareness among parents and healthcare professionals about the risk of children gaining body weight related to the life changes imposed by this long-lasting COVID-19 pandemic. Parent and child-based educational interventions, reset in this epidemiological context could positively influence children's behavior to resort to eating comfort before developing resistance to change and improve the recognition and management of their nutritional status. Future research is needed to identify new educational strategies and school-based interventions for both children and parents, targeting an optimal and more active lifestyle to prevent childhood obesity.

Conflict of interest: none declared Financial support: none declared

\section{REFERENCES}

1. Klein JD, Koletzko B, El-Shabrawi MH, et al. Promoting and supporting children's health and healthcare during COVID-19: International Paediatric Association Position Statement. Arch Dis Child. 2020; 105(7):620-4.

2. Dong Y, Mo X, Hu Y, et al. Epidemiology of COVID-19 among children in China. Pediatrics. 2020;145(6):e20200702.

3. Akseer N, Kandru G, Keats EC, et al. COVID-19 pandemic and mitigation strategies: implications for maternal and child health and nutrition. Am J Clin Nutr. 2020;112(2):251-6.
4. Koletzko B, Holzapfel C, Schneider U, et al. Lifestyle and Body Weight Consequences of the COVID-19 Pandemic in Children: Increasing Disparity. Ann Nutr Metab. 2021;77(1):1-3.

5. Guthold R, Stevens GA, Riley LM, et al. Global trends in insufficient physical activity among adolescents: A pooled analysis of 298 population-based surveys with $1 \cdot 6$ million participants. Lancet Child Adolesc Health. 2020;4:23-35.

6. Rezaeipour M. COVID-19-Related Weight Gain in School-Aged Children. Int J Endocrinol Metab. 2020;19(1):e110634.
7. Sidor A, Rzymski P. Dietary choices and habits during COVID-19 lockdown: experience from Poland. Nutrients. 2020;12(6):1657.

8. Pop TI, Maniu D, Rajka D, et al. Prevalence of Underweight, Overweight and Obesity in School-Aged Children in the Urban Area of the Northwestern Part of Romania. Int J Env Res Publ Health. 2021;18(10):5176.

9. Brooks C, Spencer JR, Sprafkaa M, et al. Pediatric BMI changes during COVID-19 pandemic: An electronic health record-based retrospective cohort study. EClinical Medicine 2021;101026. 
10. Adams EL, et al. Longitudinal associations between child weight change, the home food environment and child feeding practices during COVID-19. Presented at: American Society of Nutrition Scientific Sessions and Annual Meeting; June 7-10, 2021 (virtual meeting).

11. Vinker-Shuster M, Grossman ES, Yeshayahu Y. Increased Weight Gain of Children during the COVID-19 Lockdown. Isr Med Assoc J. 2021;23(4):219-222.

12. Lotrean LM, Popa I, Florea M, et al. Actual Weight, Perceived Weight and Desired Weight of Romanian School Children by Parents and Children. Medicina. 2021; $57: 333$.

13. Valea A, Silaghi A, Ghervan C, et al. Morbid child obesity with possible RohhadnetRohhad syndrome. Acta Endocrinologica (Buc). 2014;10(3):515-524.

14. Lotrean L, Popa M, Santillan E, et al. Methodological challenges in research regarding the lifestyle of school children. Revista de Cercetare si Interventie Sociala. 2014;44:321-331.

15. Lazea C, Sur L, Florea M. ROHHAD (Rapid-onset Obesity with Hypoventilation, Hypothalamic Dysfunction, Autonomic Dysregulation) Syndrome - What Every Pediatrician Should Know About the Etiopathogenesis, Diagnosis and Treatment: A Review. Int J Gen Med. 2021;14:319-326.
16. Dunton GF, Do B, Wang SD. Early effects of the COVID-19 pandemic on physical activity and sedentary behavior in children living in the U.S. BMC Public Health. 2020; 20(1):1351.

17. Skerritt J, Mulvay L, Almeida I. Americans drop kale and quinoa to lock down with chips and oreos. Bloomberg News; 2020. Available at: https://www.bloomberg.com/ news/articles/2020-03-21/americans-dropkale-and-quinoa-to-lock-down-with-chipsand-oreos.

18. Azizi-Soleiman F, LMotlagh ME, Qorbani M, et al. Dietary habits and health related behaviors in Iranian children and adolescents: the CASPIAN-IV study. Int J Pediatr. 2016;4(7):2087-97.

19. Ghobadi S, Totosy de Zepetnek JO, et al. Association between overweight/obesity and eating habits while watching television among primary-school children in the city of Shiraz, Iran. Public Health Nutr. 2018; 21(3):571-9.

20. Mazur A, Caroli M, Radziewicz-Winnicki I, et al. Reviewing and addressing the link between mass media and the increase in obesity among European children: The European Academy of Paediatrics (EAP) and The European Childhood Obesity Group (ECOG) consensus statement. Acta Paediatr. 2018;107(4):568-76.
21. Rundle AG, Factor-Litvak P, Suglia SF, et al. Tracking of Obesity in Childhood into Adulthood: Effects on Body Mass Index and Fat Mass Index at Age 50. Child Obes. 2020;16(3):226-33.

22. World Health Organization. Population based approaches to childhood obesity prevention. Geneve: WHO, 2012.

23. Mahan LK, Escott-Stump S, Raymond JL. Krause's Food and the Nutrition Care Process. Elsevier Saunders, 2012.

24. Team G. 10 Bodyweight Exercises You Can Do Anywhere. Power. 2019;30.

25. Rosenberg DE, Bull FC, Marshall AL, et al. Assessment of sedentary behavior with the International Physical Activity Questionnaire. Journal of Physical Activity \& Health, 2008; 5:S30.

26. Street TD, Lacey SJ, Langdon RR. Gaming Your Way to Health: A Systematic Review of Exergaming Programs to Increase Health and Exercise Behaviors in Adults. Games Health J. 2017;6(3):136-46.

27. Florea M, Lazea C, Gaga R, et al. Lights and shadows of the perception of the use of telemedicine by Romanian family doctors during the COVID-19 pandemic. Int J Gen Med. 2021;14:1576-1587.

28. Gordon WJ, Landman A, Zhang H, et al. Beyond validation: getting health apps into clinical practice. NPJ Digit Med. 2020;3:14. 